\title{
Forme verbali alternative (regolari e irregolari) del passato remoto
}

\author{
Daniel Stapek \\ Università di Breslavia \\ daniel.slapek@uwr.edu.pl
}

Za glagolski čas passato remoto je značilno, da ima veliko alternativnih oblik in to ne samo zaradi dvojnih končnic druge konjugacije. Posledično je v ro spregatvenih tabelah in slovarjih glagolov, ki so bili objavljeni $v$ Italiji kar 38 takih, ki izbirajo med dvema ali celo tremi oblikami v tem glagolskem času. Namen članka je I) dokazati, da obstajajo včasih tudi velike razlike v spregatvenih tabelah, ki predstavljajo glagolski čas passato remoto; 2) preveriti dejansko uporabo alternativnih oblik in torej njihovo leksikalno pojavnost v najbolj poznanih jezikovnih korpusih pisane italijanščine in (3) predstaviti preference maternih govorcev sodobne italijanščine pri izbiri alternativnih oblik.

Ključne besede: passato remoto, spreganje, glagolska spregatev, italijanska slovnica, sodobna italijanščina

Il passato remoto si caratterizza per un' insolita abbondanza di forme verbali alternative, non solo quelle dovute alle doppie desinenze regolari della seconda coniugazione. In tal modo, in io tavole di coniugazione e dizionari dei verbi pubblicati in Italia troviamo 38 verbi che registrano due o persino tre forme flesse (regolari e irregolari). Con il presente lavoro si vuole i) dimostrare le divergenze, a volte anche notevoli, nella presentazione della flessione del Passato Remoto nelle tavole di coniugazione; 2 ) verificare l'uso effettivo delle forme alternative, quindi le loro occorrenze lessicali nei più noti corpora d'italiano scritto; e 3) le attuali preferenze nella scelta delle forme alternative tra i parlanti di madrelingua italiana. Parole chiave: passato remoto, coniugazione, flessione verbale, grammatica italiana, italiano contemporaneo

I

sistema verbale italiano rappresenta uno degli aspetti grammaticali più problematici che il parlante - non solo apprendente straniero deve affrontare. Infatti, su vari siti di consulenza linguistica o nei più recenti volumi che trattano di dubbi linguistici non mancano quesiti relativi alle forme verbali, per così dire, più difficoltose ${ }^{\mathrm{I}}$. Sarà forse questo il motivo per cui sul mercato editoriale sono apparse varie tavole di coniugazione che, visto il loro numero, godono di un certo successo tra lettori. Sono anche questi, oltre ai

I Si vedano p. es. Silverio Novelli, Si dice? Non si dice? Dipende (Roma-Bari: Laterza, 2015); Valeria della Valle, and Giuseppe Patota, Ciliegie o ciliege? (Milano: Sperling \& Kupfer, 2012) o il sito web della consulenza linguistica dell'Accademia della Crusca. dizionari, i sussidi didattici cui si ricorre di solito in caso di emergenza. (Nella tabella I riporto l'elenco di dieci tavole e dizionari dei verbi pubblicati in Italia cui farò riferimento nel presente testo $^{2}$, per motivi di spazio, con l'abbreviazione TC indicherò il rispettivo titolo accompagnato dal numero attribuitogli nell'elenco).

Tra le caratteristiche del sistema verbale italiano troviamo la coesistenza di forme flesse alternative. I titoli citati in seguito discordano a questo proposito, a volte in maniera notevole: al-

2 Chiaramente, la lista che si propone in questo lavoro non è esaustiva, ma lo stesso numero può sempre stupire, perché il contenuto di tali testi in realtà non dovrebbe variare, tranne che - al limite - per il numero di voci comprese. 
cuni prendono in considerazione una sola forma, altri riportano due o tre forme equivalenti, altri ancora registrano forme piuttosto arcaiche (di solito senza un'apposita nota di spiegazione). In tal modo, probabilmente per tutti i tempi e modi verbali troveremo esempi di una simile alternanza flessiva, ad esempio:

I) al presente indicativo: apparire: appaio/ apparisco ( $\mathrm{TC}_{3}$, p. 90), chiedere: chiedo/ chieggo ( $\mathrm{TC}_{5}, \mathrm{p} .22$ ), possedere: possiedo/ posseggo (TC6, p. 270);

2) al futuro indicativo: udire: udrò/udirò (TC9, p. 83), soddisfare: soddisfarò/soddisferò, cogliere: coglierò/corrò (TC8, p. IO4, $\mathrm{I}_{4} 8$ ) o andare: andrò/anderò ( $\mathrm{TC}_{4}$, p. 29, però l'autore registra solo la forma andrei del condizionale semplice, che dovrebbe riprendere la radice del futuro);

3) al congiuntivo presente: dovere: deva/debba (TCI, p. 86), parere: paia/para (TC8, p. 263) o dolersi: doliamo/dogliamo (TC6, p. I52, ma al presente indicativo, anche se le due forme dovrebbero coincidere, gli autori registrano solo la prima);

4) all'imperativo: fare: fa'/fai (TC6, p. I80), andare: va'/vai $\left(\mathrm{TC}_{7}, \mathrm{p} .37\right)$, dare: da'/dai (TC6, p. 104)

5) al participio passato: propendere: propeso/ propenso/propenduto, provvedere: provvisto/provveduto, seppellire: sepolto/seppellito (TC6, pp. 293, 301, 372), e addirittura

6) al participio presente: potere: potente/possente (il secondo registrato solo in TC5, p. 24, annotato come "raro") ecc. Possono inoltre coesistere le forme che variano

a) l'apertura della vocale, come p. es. dèvo/ dévo (TC8, p. 194),

3 Alcune forme dell'imperativo della seconda persona singolare costituiscono un gruppo piuttosto particolare, perché vengono illustrate con: a) una sola forma troncata, p.es. fa' (TC 5, p. 23), di' (TC6 p. 180), o accentata: di (TC5, p. 23); b) con due forme, p.es. fa'/fai, $\mathrm{vai} / \mathrm{va}$ ' ecc. (come sopra), c) con tre rispettive forme flesse, p.es. fa/ fai/fa' (TC2, p. 80), vai/va/va'e di/di'/di (TC8, pp. 97,413), dai/da'/dà (TCio, p. 14). b) il dittongamento nelle particolari forme flesse: gioco/giuoco (TCio, p. I2),

c) il raddoppiamento consonantico: tacere: taciamo/tacciamo (TC8, p. 364; l'autrice ammette la doppia consonante solo per la prima persona plurale del presente indicativo), o

d) l'ortografia: spegnere: spegniamo/spegnamo (la possibile perdita della i non viene notata da nessuno dei dieci titoli presi in esame).

Come vediamo, una simile alternanza verbale in italiano non è un fenomeno raro, anche se alcune delle forme indicate sopra potranno stupire gli apprendenti/insegnanti stranieri (a quanto pare stupiscono anche il correttore automatico del più diffuso editore del testo) perché le grammatiche a loro destinate non ne hanno mai parlato. In particolar modo, il Passato Remoto (nel seguito abbreviato come PR) è il tempo verbale che si distingue all'interno del sistema verbale italiano per un'insolita abbondanza di forme flesse alternative. Con il presente lavoro intendo, quindi, verificare:

I) come vengono presentate le doppie, o addirittura triple, forme (regolari e irregolari) del PR nelle tavole di coniugazione o nei dizionari dei verbi italiani,

2) quali forme sono preferite nei testi scritti (vedremo l'impiego di tali forme flesse nei più grandi corpora di italiano scritto), e

3) quali forme sono preferite oggi dai parlanti di madrelingua italiana

Tabella r: Alcune tavole di coniugazione e dizionari dei verbi pubblicati in Italia

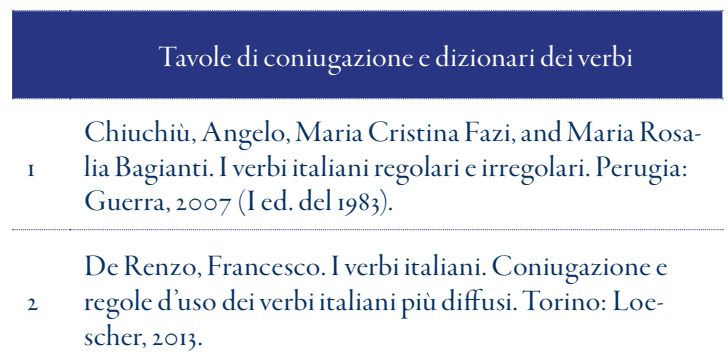


Tavole di coniugazione e dizionari dei verbi

Duse, Giorgio. Verbi italiani. Regolari, irregolari, difetti-

vi. Sesto San Giovanni: Edizioni Bignami, 2009

Elia, Pietro. I verbi italiani ad uso degli stranieri. Mila-

4 no: Edizioni Scolastiche Mondadori, 1965 (VIII edizione; I ed. del 1955).

Fava, Susanna, and Lisa Puglia. Verbi italiani. Milano: La Spiga, 2005 .

Ilardi, Francesca, and Max Bocchiola. I verbi italiani. 452

6 verbi irregolari interamente coniugati. Milano: Hoepli, 2015 (I ed. del 2006)

Manella, Claudio, and Cesare Pallante. Guida ai verbi italiani. 9a edizione. I verbi della lingua italiana regolari e

7 irregolari. Coniugazioni complete. Traduzioni in inglese, francese, tedesco, spagnolo. Esercizi e chiavi. Firenze: Edizioni Progetto Lingua, 2015 (I ed. del 1998)

Parodi, Simonetta. Dizionario. Verbi (Edizione aggiornata. Il verbo e le sue strutture generali, classificazio-

8 ne dei verbi, coniugazione dei verbi regolari e irregolari, i verbi irregolari, composti e derivati). Santarcangelo di Romagna: RusconiLibri, 2011

Reza Mousavi, Hamid. Repertorio dei verbi italiani. Firenze: Le Monnier, 2002.

Stoppelli, Marina. Verbi. Italiano (collana: Guide linguistiche). Novara: Garzanti Linguistica, 2015 (I ed. del 2004)

Doppie forme del Passato Remoto nelle tavole di coniugazione e dizionari dei verbi

La tabella 2 presenta i risultati dell'analisi delle tavole di coniugazione e dei dizionari dei verbi italiani: la prima colonna indica in ordine alfabetico tutti i verbi le cui forme del PR sono presentate come doppie (regolari e irregolari) almeno in uno dei testi esaminati; nella seconda colonna i loro paradigmi flessivi ritrovati nelle tavole (per motivi di spazio riporto qui solo la forma flessa della prima persona singolare); nelle colonne successive i numeri indicano quali (o quale) delle forme flesse della seconda colonna vengono citate da un dato testo. Inoltre,

I) con un trattino si segnala la mancanza del verbo nel rispettivo titolo;
2) i numeri tra parentesi tonde indicano le forme flesse del dato verbo ricostruite secondo le indicazioni ritrovate nell'indice dei verbi (o in diverse note degli autori), p. es. $\mathrm{TC}_{4}$ non dà una tavola di coniugazione complessiva di profferire, ${ }^{4}$ ma rimanda nell' indice a finire, per cui le forme ricostruite sono profferii, profferì, profferirono;

3) l'asterisco indica alcune irregolarità nella presentazione del paradigma flessivo di un dato verbo, p. es. TC8 per il verbo offrire registra i paradigmi offrii/offersi, ma nella terza persona del plurale indica solo offersero. Tutte le irregolarità sono spiegate in basso alla tabella.

Non entrano nell'analisi i verbi che hanno doppie forme dovute al dittongamento, come muovere: mossi, muovesti/movesti o nuocere: nocqui, nocesti/nuocesti ecc. (la prima persona singolare e la terza persona del singolare e del plurale hanno una sola forma), i verbi dare e parere, che hanno due forme irregolari (diedi/detti; parvi/ parsi), i verbi della seconda coniugazione con le doppie desinenze regolari, come temere: temei/ temetti, credere: credei/credetti ecc. ${ }^{5}$, nonché i verbi che cambiano il paradigma flessivo a seconda del significato:

I) riflettere; la dipendenza tra la forma verbale e l'accezione del verbo viene notata solo da tre titoli: "riflettere nel significato di 'pensare' adotta il participio riflettuto, invece nel significato di 'rispecchiare' il participio riflesso", TCro, p. 48; similmente in $\mathrm{TC}_{9}$, p. 24, cfr. $\mathrm{TC}_{7}$, p. 228 ; $^{6}$ visto che le forme del Participio Passato e del Passato Remoto sono interdipendenti, è facile dedurne la forma del PR (TC8 a p. 310 riporta solo il paradigma riflettei);

4 Citato anche come proferire, quindi con una sola $\mathrm{f}$.

5 In unaltra sede esamino in dettaglio il problema delle doppie desinenze regolari; si veda Daniel Stapek, «Doppie forme regolari del Passato Remoto: desinenze -ei, -è, - erono vs -etti, -ette, -ettero», to appear.

6 La dicitura di $\mathrm{TC}_{7}$ è ambigua: "riflettere nel significato di to reflect [...] ha il participio passato irregolare riflesso e al Passato Remoto, oltre alla forma regolare, c'è anche la forma io riflessi"; "anche" suggerisce in questo caso l'equivalenza delle due forme. 
2) inferire: "nel senso di 'dare, colpire' al PR presenta le forme irregolari infersi, inferse, infersero [...]; nel senso di 'dedurre, desumere' al PR ha le forme regolari inferii, inferisti, inferi" (TCio, p. 98; la differenza notata solo da questo titolo); 3 ) succedere: successe nel senso di 'accadere' e successe/succedette (accettato anche succedé) nel senso di 'subentrare' (“Augusto, primo im- peratore romano, successe (o succedette) $\mathrm{Ti}$ berio"; esempio tratto da Treccani); nessuna delle tavole distingue i due significati; $\mathrm{TC}_{7}$ riporta solo successe in quanto verbo difettivo, quindi esclude del tutto la seconda accezione.

Per i verbi che possono avere due forme flesse, $\mathrm{A}$ (con una desinenza regolare) e B (forma irregolare), ritroviamo diverse combinazioni, tra le quali:

Tabella 2: Forme alternative (regolari e irregolari) del PR registrate nelle tavole di coniugazione

\begin{tabular}{|c|c|c|c|c|c|c|c|c|c|c|c|}
\hline & & $\mathrm{TC}_{\mathrm{I}}$ & $\mathrm{TC}_{2}$ & $\mathrm{TC}_{3}$ & $\mathrm{TC}_{4}$ & $\mathrm{TC}_{5}$ & $\mathrm{TC} 6$ & $\mathrm{TC}_{7}$ & TC8 & $\mathrm{TC}_{9}$ & TCro \\
\hline annettere & annettei/annessi & 2 & - & 2 & $\mathrm{I} / 2$ & $\mathrm{I} / 2$ & $\mathrm{I} / 2$ & - & $1 / 2$ & - & $(\mathrm{I} / 2)$ \\
\hline apparire & apparii/apparsi/apparvi & $(\mathrm{I} / 2 / 3)$ & - & $1 / 2 / 3$ & $1 / 2 / 3$ & $1 / 2 / 3$ & 3 & $(\mathrm{I} / 2 / 3)$ & 3 & 3 & 3 \\
\hline aprire & aprii/apersi & I & I & $\mathrm{I} / 2$ & $1 / 2$ & $1 / 2$ & $\mathrm{I} / 2$ & I & $\mathrm{I} / 2$ & $1 / 2$ & $\mathrm{I} / 2$ \\
\hline assolvere & assolvei/assolvetti/assolsi & (3) & - & 3 & 3 & 3 & 3 & (3) & 3 & $\mathrm{I} / 2 / 3$ & 3 \\
\hline bere & bevei/bevetti/bevvi & 3 & $2 / 3$ & $2 / 3$ & 3 & $2 / 3$ & $2 / 3$ & 3 & $1 / 2 / 3$ & $2 / 3$ & 3 \\
\hline cadere & cedetti/caddi & 2 & 2 & 2 & 2 & 2 & 2 & 2 & $1 / 2^{*}$ & 2 & 2 \\
\hline comparire & $\begin{array}{l}\text { comparii/comparsi/com- } \\
\text { parvi }\end{array}$ & $1 / 2 / 3$ & - & $(\mathrm{I} / 2 / 3)$ & $(\mathrm{I} / 2 / 3)$ & $(\mathrm{I} / 2 / 3)$ & - & $1 / 2 / 3$ & - & (3) & (3) \\
\hline concedere & $\begin{array}{l}\text { concedei/concedetti/con- } \\
\text { cessi }\end{array}$ & 3 & - & 3 & $1 / 2 / 3^{*}$ & 3 & $2 / 3$ & 3 & $1 / 2 / 3^{*}$ & $1 / 2 / 3$ & 3 \\
\hline connettere & connettei/connessi & (2) & - & $(2)$ & $(\mathrm{I} / 2)$ & $(\mathrm{I} / 2)$ & $1 / 2$ & - & - & I & $(\mathrm{I} / 2)$ \\
\hline coprire & coprii/copersi & I & I & $1 / 2$ & $(\mathrm{I} / 2)$ & $1 / 2$ & $1 / 2^{*}$ & I & I & $(2)$ & $(\mathrm{I} / 2)$ \\
\hline costruire & costruii/costrussi & (I) & I & ( & - & $1 / 2$ & - & I & I & - & $(\mathrm{I})$ \\
\hline deflettere & deflettei/deflessi & - & - & $(2)$ & $(\mathrm{I} / 2)$ & (2) & $1 / 2$ & - & - & - & $(\mathrm{I} / 2)$ \\
\hline devolvere & devolvei/devolvetti/devolsi & 3 & - & - & - & (3) & $1 / 2$ & - & $1 / 3^{*}$ & - & $(3)$ \\
\hline discutere & discutei/discussi & 2 & 2 & 2 & $1 / 2$ & 2 & - & 2 & 2 & 2 & 2 \\
\hline flettere & flettei/flessi & - & - & 2 & $(\mathrm{I} / 2)$ & 2 & $\mathrm{I} / 2$ & - & - & - & $\mathrm{I} / 2$ \\
\hline genuflettersi & genuflettei/genuflessi & - & - & - & $(\mathrm{I} / 2)$ & - & $1 / 2^{*}$ & - & - & - & $(\mathrm{I} / 2)$ \\
\hline offrire & offrii/offersi & I & I & $\mathrm{I} / 2$ & $(\mathrm{I} / 2)$ & $1 / 2$ & $\mathrm{I} / 2$ & I & $1 / 2^{*}$ & $(2)$ & $(\mathrm{r} / 2)$ \\
\hline perdere & perdei/perdetti/persi & 3 & $1 / 2 / 3$ & 3 & $1 / 2 / 3$ & 3 & $\mathrm{I} / 2 / 3^{*}$ & 3 & $1 / 2 / 3^{*}$ & $1 / 2 / 3$ & 3 \\
\hline prescindere & $\begin{array}{l}\text { prescindei/prescindetti/ } \\
\text { prescissi }\end{array}$ & (3) & - & - & (3) & - & $1 / 2 / 3^{*}$ & (3) & - & - & (3) \\
\hline $\operatorname{prof}(f)$ erire & $\operatorname{prof}(f) \operatorname{erii} / \operatorname{prof}(f)$ ersi & (I) & - & ( I) & - & $1 / 2$ & - & - & - & - & (I) \\
\hline propendere & propendei/propesi & - & - & - & - & - & $1 / 2$ & - & - & - & - \\
\hline redigere & redigei/redassi & 2 & - & - & $1 / 2$ & - & 2 & 2 & $1 / 2^{*}$ & - & 2 \\
\hline rendere & rendei/resi & 2 & - & 2 & (2) & 2 & 2 & 2 & $1 / 2^{*}$ & $(2)$ & (2) \\
\hline retrocedere & $\begin{array}{l}\text { retrocedei/retrocedetti/ } \\
\text { retrocessi }\end{array}$ & $2 / 3$ & - & (3) & $(\mathrm{I} / 3)$ & (3) & - & (3) & 3 & $(\mathrm{I} / 2 / 3)$ & (3) \\
\hline riannettere & riannettei/riannessi & - & - & - & - & - & $1 / 2$ & - & - & - & $(\mathrm{I} / 2)$ \\
\hline
\end{tabular}




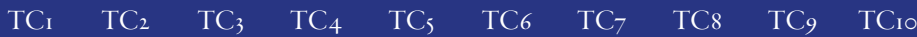

\begin{tabular}{|c|c|c|c|c|c|c|c|c|c|c|c|}
\hline riconnettere & riconnettei/riconnessi & - & - & - & - & - & $1 / 2$ & - & - & - & $(\mathrm{I} / 2)$ \\
\hline risolvere & risolvetti/risolsi & 2 & 2 & $(2)$ & (2) & (2) & 2 & 2 & $1 / 2^{*}$ & - & (2) \\
\hline scernere & scernei/sernetti/scersi & - & - & $(\mathrm{I} / 2)$ & - & - & - & - & $1 / 2 / 3^{*}$ & - & - \\
\hline sconnettere & sconnettei/sconnessi & (2) & - & (2) & $(\mathrm{I} / 2)$ & $(\mathrm{I} / 2)$ & $1 / 2$ & - & - & - & $(\mathrm{I} / 2)$ \\
\hline scoprire & scoprii/scopersi & I & (I) & $(\mathrm{I} / 2)$ & $(\mathrm{I} / 2)$ & $(\mathrm{I} / 2)$ & $1 / 2^{*}$ & (I) & - & $(\mathrm{I} / 2)$ & $(\mathrm{I} / 2)$ \\
\hline soffrire & soffrii/soffersi & I & $\mathrm{I} / 2$ & $1 / 2$ & $(\mathrm{I} / 2)$ & $1 / 2$ & $\mathrm{I} / 2$ & I & - & $(\mathrm{I} / 2)$ & $(\mathrm{I} / 2)$ \\
\hline spandere & spandei/spansi & - & - & - & * & - & $1 / 2^{*}$ & - & $1 / 2^{*}$ & (2) & (2) \\
\hline sparire & sparii/sparvi & (I) & - & $(\mathrm{I})$ & - & $1 / 2$ & - & (I) & $1 / 2^{*}$ & (I) & (2) \\
\hline tacere & tacetti/tacqui & 2 & - & 2 & (2) & 2 & 2 & 2 & $1 / 2^{*}$ & (2) & (2) \\
\hline tenere & tenei/tenni & 2 & 2 & 2 & 2 & 2 & 2 & 2 & 2 & 2 & $I / 2$ \\
\hline vedere & vedei/vedetti/vidi & 3 & 3 & 3 & 3 & 3 & 3 & 3 & $1 / 2 / 3^{*}$ & 3 & 3 \\
\hline
\end{tabular}

Particolarità:

TC4: concedere: concedei/concessi, concedé/cocesse, concedettero/concessero; spandere: (spandei/spandetti, spandé/ spandette, spanderono/spandettero)

apparire: apparvi; coprire: coprii/copersi, coprì, coprirono; genuflettersi: mi genuflettei/genuflessi, si genufletté/genuflesse, si genuflessero; perdere: persi/perdei, perse/perdette, persero/perdettero; prescindere: prescindei/prescindetti/(raro)prescissi, prescindé/prescisse, prescinderono/prescissero; scoprire: scoprii/scopersi, scoprì, scoprirono; spandere: spandei/spansi, spandé/spanse, spansero

cadere: caddi, cadde/cadette, caddero; concedere: concessi/concedei/concedetti, concesse, concessero; devolvere: devolvei/devolsi, devolse, devolsero; offrire: offrii/offersi, offri/offerse, offersero; perdere: perdei/perdetti/persi, per-

TC8: se, persero; redigere: redigei/redassi, redasse, redassero; rendere: rendei/resi, rese, resero; risolvere: risolvetti/risolsi, risolvette/risolse, risolsero; scernere: scernei/scernetti/scersi, scerse, scersero; spandere: spandei, spanse, spansero; sparire: sparii, spari/sparve, sprirono/sparvero; tacere: tacqui/tacetti, tacque, taquero; vedere: vedei/vedetti/vidi, vedette/vide, videro

I) forme equivalenti $\mathrm{A} / \mathrm{B}$ o solo la forma $\mathrm{A}$, dove sul totale dei testi analizzati prevale A/B, p. es. soffrire, scoprire, aprire;

2) $\mathrm{A} / \mathrm{B}$ o $\mathrm{A}$ (dove prevale $\mathrm{A})$ : profferire, costruire;

3) $\mathrm{A} / \mathrm{B}$ (prevalente) o B: sconnettere, annettere, deflettere;

4) $\mathrm{A} / \mathrm{B}$ o B (prevalente): tenere, tacere, redigere;

5) AB o A o B: sparire, connettere, coprire, offrire;

6) solo A/B: riconnettere, riannettere (indicate expressis verbis in una sola tavola), genuflettersi (riportato solo da una tavola, in altre due le sue forme flesse sono ricostruite). Cambiano inoltre le desinenze regolari per i verbi della seconda coniugazione; troveremo quindi le desinenze brevi -ei, -é, -ero- no (nella maggior parte dei verbi sopra citati, p. es. rendé, redigé ecc.) o lunghe -etti, -ette -ettero (in tacette, risolvette e cadette).

La maggior parte dei verbi che hanno tre forme sono verbi uscenti in -ere che, oltre alle due forme create per aggiunta di due desinenze regolari (come sopra), hanno una forma flessa irregolare

a) riconducibile alla forma del Passato Prossimo (scernere: scersi, perdere: persi, retrocedere: retrocessi, devolvere: devolsi, assolvere: assolsi, prescindere: prescissi, concedere: concessi) o

b) del tutto irregolare (bere: bevvi, vedere: vidi). Prevalgono solitamente le forme irregolari. Tra i verbi con le triple forme del PR troviamo anche due verbi in -ire: apparire (apparii/apparsi/apparvi) e - similmente - 
comparire (comparii/comparsi/comparvi); in questo caso le tre forme flesse sono nella maggior parte dei titoli indicate come equivalenti.

\section{Passato Remoto (ir)regolare nei corpora d'italiano scritto}

L'analisi del numero di occorrenze del PR è stata effettuata sui più noti corpora di italiano scritto: ${ }^{7}$

I) CORIS, che contiene 130 milioni di parole ed è "costituito da una raccolta di testi, autentici e ricorrenti nell'uso, in formato elettronico, selezionati come rappresentativi dell'italiano attuale";

2 Italian Web 2016 (itTenTenı6; nel seguito abbreviato come ItWeb), componente del sistema SketchEngine che raccoglie testi tratti da Internet; oggi la più grande risorsa lessicale per la lingua italiana con quasi 5 miliardi di parole;

3) "la Repubblica", quindi un corpus composto dagli articoli del giornale pubblicati tra il 1985 e il 2000 , con pressoché 380 milioni di parole;

4) PAISÀ, di 250 milioni di parole, contiene testi raccolti dal web nel settembre/ottobre del 20ro; "la raccolta di testi contemporanei è stata creata [...] allo scopo di fornire materiale autentico e disponibile gratuitamente per l'apprendimento dell'italiano";

5) CoLfIS (Corpus e Lessico di Frequenza dell'Italiano Scritto), quindi "un corpus bilanciato di oltre 3 milioni di parole, che rispecchia le tendenze di lettura degli italiani desunte da dati ISTAT”. Il lemmario del

7 Per una panoramica delle risorse lessicali in italiano si veda p. es. Emanuela Cresti, Alessandro Panunzi, Introduzione ai corpora dell'italiano (Bologna: il Mulino, 2013), I4I-154.

8 Tutte le citazioni relative ai corpora sono tratte dai rispettivi siti web dei singoli progetti: CORIS: http:/corpora.dslo.unibo.it/ coris_ita.html; PAISÀ: http://www.corpusitaliano.it; CoLFIS: http://linguistica.sns.it/CoLFIS/Home.htm.
CoLFIS servirà inoltre per ordinare i verbi nella sezione $4 .{ }^{9}$

I numeri di occorrenze sono disposti in due tabelle: la tabella 3 prende in considerazione i verbi che nelle tavole di coniugazione hanno registrato due forme alternative del PR, la tabella 4 contiene i verbi con tre forme flesse. I verbi, a loro volta, sono disposti in ordine crescente a seconda del numero complessivo delle loro occorrenze del PR nei cinque corpora analizzati. Siccome alcune voci del PR coincidono con le forme del Participio Passato (p. es. annesse, connesse ecc.), sono state analizzate le forme della terza persona plurale, prive di tale ambiguità sintattica.

I verbi che hanno le doppie forme flesse sono stati divisi in due gruppi, in cui a) prevalgono le forme flesse regolari, e b) prevalgono le forme flesse irregolari. In tal modo, nel primo gruppo entrano tutti i verbi uscenti in -ire, insieme a tre verbi in -ere: flettere, deflettere, riannettere (il cui numero complessivo nei cinque corpora è molto limitato, perciò non sarebbe lecito dedurne una regola grammaticale; si paragonino nel seguito i numeri relativi a questi verbi che risultano dal questionario). Per gli altri verbi in -ere si registrano invece più numerose le forme irregolari.

Nel corpus ItWeb, dove le forme del PR sono più diffuse rispetto ad altri corpora, le forme prevalenti costituiscono una percentuale relativamente molto alta di tutte le occorrenze del PR (mi limito a elencare i verbi le cui forme del PR superano la soglia di 100 occorrenze): per le forme regolari: aprirono $96,7 \%$, costruirono $99,4 \%$, scoprirono $97,4 \%$, offrirono $93,7 \%$, sparirono $96,1 \%$, coprirono $96,3 \%$, soffrirono $90,7 \%$; per le forme irregolari, rispettivamente: resero $99,1 \%$, caddero $99,9 \%$, tennero $99,9 \%$, risolsero $87,9 \%$, tacquero $99,9 \%$, discussero $99,8 \%$, redassero $100 \%$.

Per quanto riguarda i verbi con tre varianti flesse, per i verbi della seconda coniugazione preval-

\footnotetext{
I motori di ricerca di It Web, CoLFIS e PAISÀ permettono di selezionare le parole annotate come verbi. Nel Coris ho posto la seguente domanda CQL: [word="X" \& pos="V_GVRB"], quindi general verb forms; nel corpus Repubblica: [word="X" \& pos="VER:fin"], quindi verb finite form.
} 
Tabella 3: Il numero di occorrenze delle forme del PR nei corpora (verbi con due forme flesse)

\section{$\begin{array}{llll}\text { CORIS } & \text { ItWeb } & \text { Repubblica } & \text { PAISA }\end{array}$}

a) prevalgono le forme flesse regolari

\begin{tabular}{|c|c|c|c|c|c|c|c|c|c|c|c|c|}
\hline aprire & aprirono & apersero & 362 & 4 & 6524 & 225 & 334 & 3 & 1077 & 3 & 6 & - \\
\hline costruire & costruirono & costrussero & 132 & - & 7299 & 46 & 189 & - & 1622 & - & 7 & - \\
\hline scoprire & scoprirono & scopersero & 259 & I & 5117 & 139 & 484 & I & 1002 & - & 8 & I \\
\hline offrire & offrirono & offersero & I3I & 4 & 3008 & 202 & 202 & 6 & 476 & 5 & I & - \\
\hline sparire & sparirono & sparvero & 107 & - & 1698 & 68 & 149 & - & 210 & - & 2 & - \\
\hline coprire & coprirono & copersero & 63 & I & 879 & 34 & 48 & - & 100 & I & 2 & - \\
\hline soffrire & soffrirono & soffersero & 14 & 2 & 753 & 77 & 30 & 2 & 182 & 2 & - & - \\
\hline $\operatorname{prof}(f)$ erire & prof $(f)$ erirono & prof(f)ersero & - & - & 53 & 14 & - & - & - & - & - & - \\
\hline flettere & fletterono & flessero & - & - & 4 & 2 & - & - & - & - & - & - \\
\hline deflettere & defletterono & deflessero & - & - & I & - & - & - & - & - & - & - \\
\hline riannettere & riannetterono & riannessero & - & - & - & - & - & - & I & - & - & - \\
\hline \multicolumn{13}{|c|}{ b) prevalgono le forme flesse irregolari } \\
\hline rendere & renderono & resero & - & 321 & 84 & 9507 & - & 433 & - & 1954 & - & 6 \\
\hline cadere & cadettero & caddero & - & 345 & II & 7978 & - & 322 & - & 1012 & - & 8 \\
\hline tenere & tenerono & tennero & - & 236 & 5 & 6068 & - & 332 & - & 1515 & - & 3 \\
\hline risolvere & risolvettero & risolsero & I & 16 & 94 & 685 & - & 46 & - & 175 & - & I \\
\hline tacere & tacettero & tacquero & - & 96 & I & $74 \mathrm{I}$ & - & 48 & - & 29 & - & 2 \\
\hline discutere & discuterono & discussero & - & 42 & I & 620 & - & 52 & - & IIO & - & 2 \\
\hline redigere & redigerono & redassero & - & 2 & - & 156 & - & 9 & - & 28 & - & - \\
\hline annettere & annetterono & annessero & I & 2 & 25 & $4 \mathrm{I}$ & - & - & 27 & 29 & - & - \\
\hline connettere & connetterono & connessero & - & - & 2 & 4 & - & - & - & 2 & - & - \\
\hline genuflettersi & genufletterono & genuflessero & - & I & - & 6 & - & - & - & - & - & - \\
\hline propendere & propenderono & propesero & - & - & - & 2 & - & - & - & 3 & - & - \\
\hline spandere & spanderono & spansero & - & - & - & 3 & - & - & - & 2 & - & - \\
\hline riconnettere & riconnetterono & riconnessero & - & - & - & I & - & - & - & - & - & - \\
\hline sconnettere & sconnetterono & sconnessero & - & - & - & I & - & - & - & - & - & - \\
\hline
\end{tabular}

gono sempre le forme irregolari; per i verbi della terza coniugazione prevalgono maggiormente le forme in -vero (nel corpus PAISÀ, invece, quelle in -irono; ciò potrebbe suggerire che la norma [o meglio: l'uso effettivo] sta cambiando, perché questo corpus raccoglie i testi più recenti). Le forme del PR dei verbi scernere e prescindere sono del tutto assenti, per cui possono considerarsi difettivi del Passato Remoto (vengono esclusi nella sezione 4).
Nel corpus ItWeb le rispettive percentuali delle forme più diffuse sono: videro $99,9 \%$, bevvero $92,3 \%$, persero $90,2 \%$, concessero $93,1 \%$, retrocessero $66,4 \%$ (retrocedettero satura una percentuale relativamente alta, pari al $28,9 \%$, assente però in tutte le altre banche dati), assolsero 93,6\%, devolsero $100 \%$, apparvero $94,8 \%$ e comparvero $94 \%$. Nel Coris le forme irregolari dei verbi in ere e le forme uscenti in -vero dei verbi della terza coniugazione coprono il Ioo\% di occorrenze, 
Tabella 4: Il numero di occorrenze delle forme del PR nei corpora (verbi con tre forme flesse)

\begin{tabular}{|c|c|c|c|c|c|c|c|c|c|c|c|c|c|c|c|c|c|c|}
\hline & & & & \multicolumn{3}{|c|}{ Coris } & \multicolumn{3}{|c|}{ ItWeb } & \multicolumn{3}{|c|}{ Repubblica } & \multicolumn{3}{|c|}{ Paisà } & \multicolumn{3}{|c|}{ Colfis } \\
\hline vedere & -erono & -ettero & videro & & & 1010 & 3 & 10 & 22624 & & - & 888 & 2 & & 3186 & & - & 20 \\
\hline bere & -erono & -ettero & bevvero & & & 100 & I & 55 & 669 & & & II & & & 30 & & - & 2 \\
\hline perdere & -erono & -ettero & persero & & 22 & 249 & 134 & 804 & 8647 & & 25 & 621 & 4 & & 2410 & & & 9 \\
\hline concedere & -erono & -ettero & - cessero & & & 37 & 13 & 69 & III3 & & & 6I & & & 250 & & & I \\
\hline retrocedere & -erono & -ettero & -cessero & & & 3 & 7 & 43 & 99 & & & 2 & & 29 & 87 & & & \\
\hline assolvere & -erono & -ettero & assolsero & & & 8 & 4 & 5 & ${ }_{131}$ & & & 28 & & - & 19 & . & - & \\
\hline devolvere & -erono & -ettero & devolsero & & & & & & 13 & & & I & & & 4 & - & - & . \\
\hline scernere & -erono & -ettero & scersero & & & & & & & & & & & - & & & & - \\
\hline prescindere & -erono & -ettero & - cissero & & - & & & & & - & - & & & & - & - & & \\
\hline apparire & -irono & -sero & -vero & - & - & 324 & 245 & 49 & 5324 & 10 & - & 300 & 130 & 8 & 62 & - & - & 7 \\
\hline comparire & -irono & -sero & -vero & - & - & ${ }_{151}$ & ${ }_{151}$ & 33 & 2882 & 5 & - & 130 & 49 & 3 & 39 & - & - & 4 \\
\hline
\end{tabular}

eccetto perdere, dove persero copre il 91,9\%. Similmente, nel Colfis, se presenti, sono registrate solo le forme irregolari (e quelle in -vero).

\section{Le preferenze nella scelta del PR dei parlanti di madrelingua italiana}

Per verificare le attuali preferenze nella scelta tra le alternative forme verbali, è stato creato un questionario on line di Google Drive ${ }^{\text {Io }}$ contenente tutti i verbi elencati nella tabella I insieme alle loro forme flesse del PR registrate nelle tavole di coniugazione (tranne prescindere e scernere, le cui forme del PR, come abbiamo visto nella tabella 4 , sono assenti nei corpora). I 34 verbi sono stati ordinati a seconda del loro rango d'uso nel CoLFIS (ciò per indicare i verbi meno frequenti - quindi poco usati, specie al PR - che potevano suscitare eventuali dubbi, nella parte finale del questionario). Inoltre, sono state usate le forme flesse della terza persona singolare, perché risultano le più frequenti nei corpora di italiano scritto.

Io Tuttora disponibile: https://goo.gl/forms/szQocjAUmxxGbsPn2.
Il questionario è stato distribuito ai parlanti di madrelingua italiana tramite vari gruppi social network e ha registrato 1282 risposte in totale. I partecipanti potevano scegliere le forme da loro preferite, quindi le forme flesse che avrebbero usato loro, potevano saltare i verbi le cui forme non avrebbero, invece, usato al PR o scegliere più forme (per cui nelle tabelle che seguono il numero totale delle singole risposte può superare il numero dei partecipanti al sondaggio, p. es. per il verbo aprire hanno risposto 1273 persone, tra cui 1264 hanno scelto aprì e 4I aperse (i numeri esatti sono: 1232 per aprì, 9 per aperse e 32 per entrambe le forme ${ }^{\mathrm{II}}$ ).

Per facilitare la lettura dei dati, come pri$\mathrm{ma}$, $\mathrm{i}$ risultati sono divisi in due tabelle relative ai verbi che a) ammettono doppie forme flesse del PR (tabella 5; le colonne i e 4 riportano le singole forme flesse, le colonne 2 e 5 : il numero di riposte per le date forme, le colonne 3 e 6 : la percentuale dei partecipanti al questionario che ha scelto data forma flessa) e b) verbi che hanno tre

I Il file in formato xls contenente tutti i dati è disponibile sul profilo academia.edu dell'autore: https://wroc.academia.edu/DanielSlapek; sezione Varia, nome del file: "Passato Remoto: alternative forme verbali (regolari e irregolari)" 
Tabella 5: Questionario: risposte relative ai verbi con doppia flessione

\begin{tabular}{|c|c|c|c|c|c|}
\hline I & 2 & 3 & 4 & 5 & 6 \\
\hline tené & 16 & $1,3 \%$ & tenne & 1264 & $99,4 \%$ \\
\hline rendé & 6 & $0,5 \%$ & rese & 1264 & $99,7 \%$ \\
\hline aprì & 1264 & $99,3 \%$ & aperse & $4 \mathrm{I}$ & $3,2 \%$ \\
\hline scoprì & 1256 & $98,9 \%$ & scoperse & 58 & $4,6 \%$ \\
\hline offrì & 1244 & $97,8 \%$ & offerse & 72 & $5,7 \%$ \\
\hline costruì & 1263 & $99,6 \%$ & costrusse & 5 & $0,4 \%$ \\
\hline cadette & 44 & $3,5 \%$ & cadde & 1255 & $98,7 \%$ \\
\hline risolvette & 170 & $13,4 \%$ & risolse & 1140 & $90,1 \%$ \\
\hline soffrì & I24I & $97,6 \%$ & sofferse & 79 & $6,2 \%$ \\
\hline discuté & 34 & $2,7 \%$ & discusse & 1252 & $98,8 \%$ \\
\hline coprì & 1251 & $98,6 \%$ & coperse & 42 & $3,3 \%$ \\
\hline sparì & 1255 & $98,9 \%$ & sparve & 40 & $3,2 \%$ \\
\hline tacette & 31 & $2,4 \%$ & tacque & 1265 & $99,4 \%$ \\
\hline redigé & 96 & $8,1 \%$ & redasse & 1093 & $92,2 \%$ \\
\hline connetté & 335 & $27,8 \%$ & connesse & 910 & $75,4 \%$ \\
\hline annetté & 351 & $29,2 \%$ & annesse & 894 & $74,4 \%$ \\
\hline spandé & 155 & $13,4 \%$ & spanse & 1016 & $87,9 \%$ \\
\hline propendé & 122 & $10,4 \%$ & propese & 1061 & $90,7 \%$ \\
\hline fletté & 250 & $20,4 \%$ & flesse & 1028 & $84, \circ \%$ \\
\hline profferì & 1025 & $83,0 \%$ & profferse & 256 & $20,7 \%$ \\
\hline sconnetté & 260 & $21,9 \%$ & sconnesse & 966 & $8 \mathrm{I}, 2 \%$ \\
\hline si genufletté & 107 & $8,5 \%$ & sigenuflesse & 1177 & $93,9 \%$ \\
\hline defletté & 243 & $21,4 \%$ & deflesse & 917 & $80,9 \%$ \\
\hline riannetté & $26 \mathrm{I}$ & $21,6 \%$ & riannesse & 992 & $82,1 \%$ \\
\hline riconnetté & 270 & $22,3 \%$ & riconnesse & 987 & $8 \mathrm{I}, 4 \%$ \\
\hline
\end{tabular}

forme del PR (tabella 6; similmente, le colonne $\mathrm{I}$, 4, 7: forme flesse; 2, 5, 8: numero di risposte; 3, 6, 9: percentuale dei partecipanti). Nella parte destra delle tabelle troviamo un grafico a barre in pila che visualizza le proporzioni il cui totale cumulativo equivale al ıoo\%.

Nella tabella 5, per i verbi in -ire si preferiscono le forme regolari (la percentuale delle risposte è molto alta e varia dal $98,9 \%$ al $99,3 \%$; l'unico verbo con una percentuale minore è profferire, profferì $83 \%$, ma è anche il verbo della terza coniugazione con il minore rango d'u-

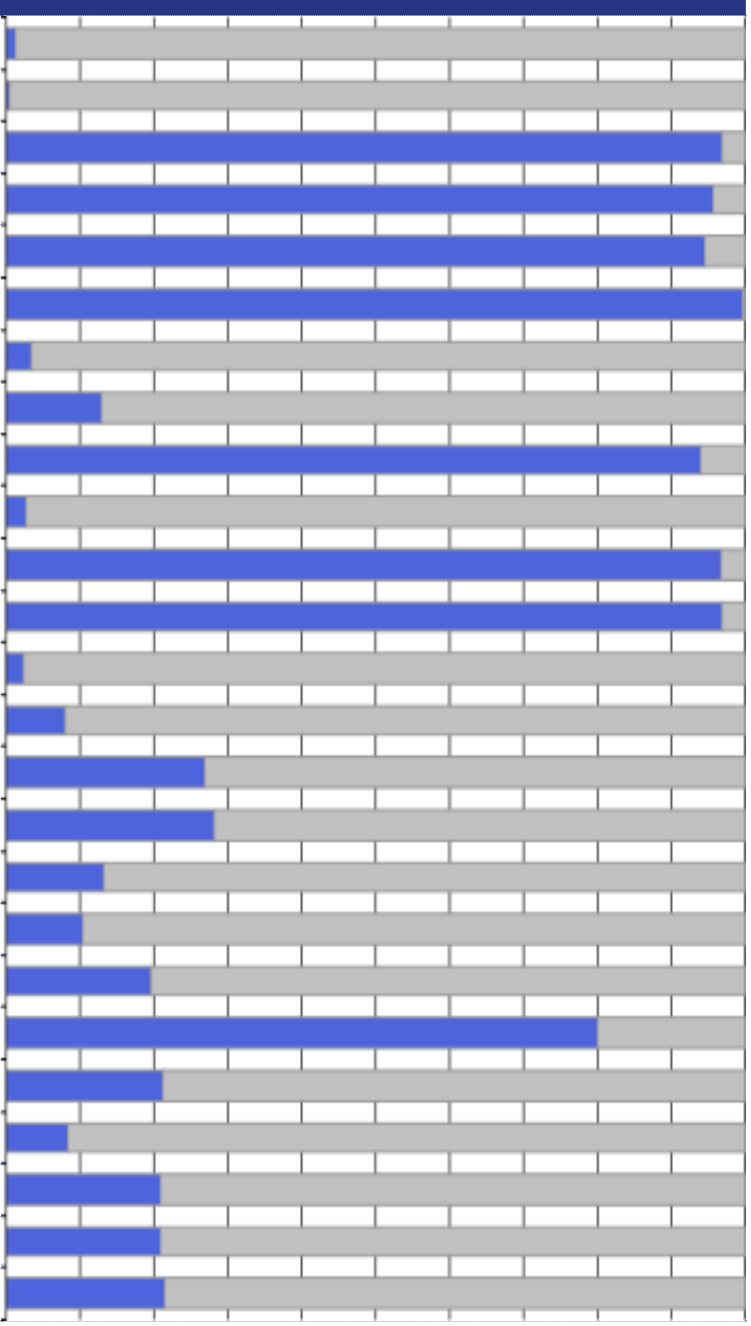

so secondo la lista di frequenza CoLFIS). ${ }^{12}$ Tutti i verbi in -ere preferiscono le forme irregolari.

Nella tabella 6, i due verbi in -ire preferiscono le forme apparve $(82,5 \%)$ e comparve $(79,2 \%)$, ma le loro desinenze regolari sono tuttavia altamente impiegate (apparì 23,6\%, comparì 25,8\%); i verbi in -ere preferiscono le forme irregolari, specie vide (96,2\%) e bevve (96,8\%), quindi verbi le cui forme del PR non dipendono dal Parti-

I2 Come risulta dai due grafici, in linea di massima per i verbi meno frequenti cresce anche il numero di risposte relative alle forme verbali meno usate. 
Tabella 6: Questionario: risposte relative ai verbi con tripla flessione

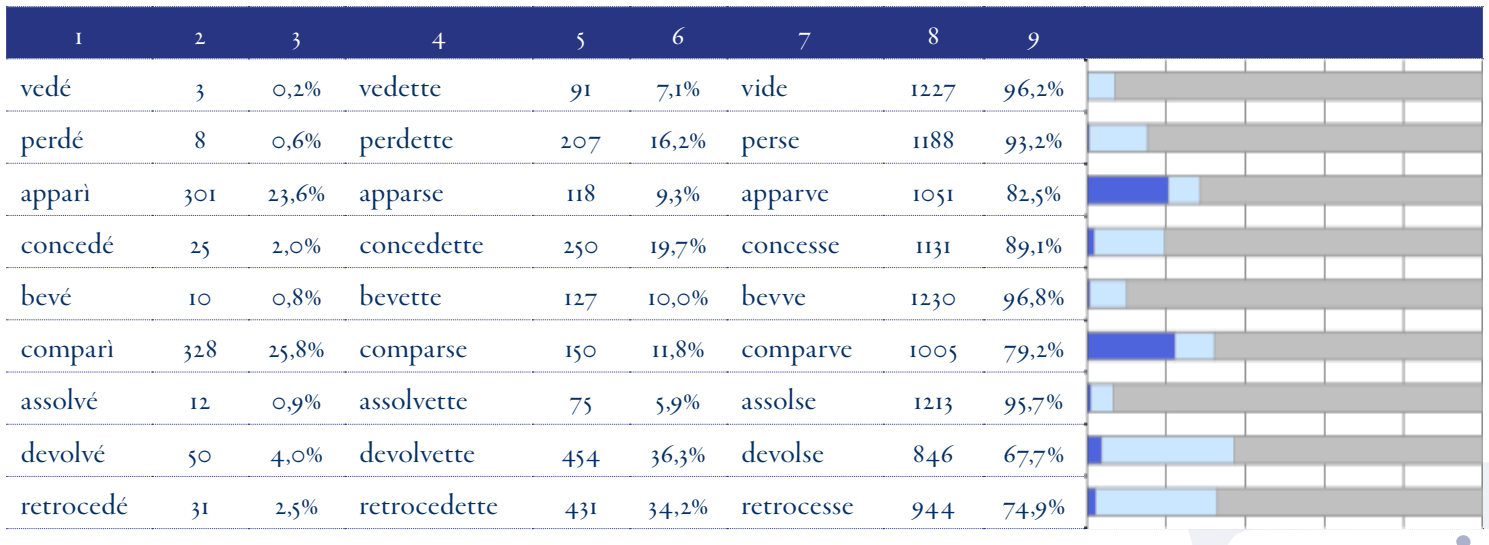

cipio Passato; sono relativamente alte le percentuali di devolvette $(36,3 \%)$ e retrocedette $(34,2 \%)$.

\section{Conclusioni}

Le risposte al questionario coincidono, in linea di massima, con i dati ricavati dallo studio sui corpora. L'unica differenza riguarda, come abbiamo già visto, i tre verbi della seconda coniugazione - flettere, deflettere, riannettere - dei quali, nei corpora, sono più numerose le forme regolari del PR (con poche effettive occorrenze lessicali, rispettivamente: $6, \mathrm{I}, \mathrm{I}$ ), invece nel questionario prevalgono le forme irregolari, con più dell' $80 \%$ di risposte (i numeri esatti sono rispettivamente: 1028, 917, 992; per cui senza dubbio possono essere considerate come preferite).

Numerose forme flesse hanno superato la soglia del 95\% di prevalenza su altre forme, sia nel corpus ItWeb sia nel questionario, tra cui: aprì (corpus: 96,7\%, questionario: 99,3\%; solo 3 tavole indicano esclusivamente questa forma, tutte le altre registrano aprì/aperse); scoprì (97,4\% e 98,9\%; nelle tavole prevalgono i doppi paradigmi flessivi); coprì ( $96,3 \%$ e $98,6 \%$; in 4 tavole registrata come unica); sparì ( $96,1 \%$ e $98,9 \%$; prevale come unica, anche se ricostruita); vide (99,9\% e 96,2\%; da 9 testi è la sola forma flessa citata). Alcune forme superano addirittura la soglia del 99\% di prevalenza: costruì (similmente, nel corpus: $99,4 \%$, nel questionario: $99,6 \%$ ), tenne $(99,9 \%, 99,4 \%)$, rese $(99,1 \%, 99,7 \%)$, cadde (99,9\%, $98,7 \%)$, discusse $(99,8 \%, 98,8 \%)$, tacque
(99,9\%, 99,4\%); per questo gruppo di verbi potremo sempre indicare un titolo che registra forme alternative (gli altri autori ammettono le forme appena citate come uniche).

Il questionario distribuito ai madrelingua finisce con una casella dedicata a "eventuali commenti". Tra i più numerosi troviamo quelli riguardanti

I) l'uso corretto o meno delle forme ivi comprese (p. es. "vorrei conoscere i miei errori" o "per alcuni verbi mi sembrano corrette due forme");

2) l'uso limitato dei verbi elencati ("alcuni verbi impossibili da sentire", "desueti o sostituiti da perifrasi", "temo di non aver mai usato il verbo scernere in vita mia”);

3) la mancata forma del PR ("molti verbi mi sembrano privi di passato remoto" o "poco probabili");

4) l'uso diversificato a seconda del contesto ("ho segnato offerse insieme ad offrì perché mi pare si usi ancora durante la Messa", "scoperse, offerse e sparve talvolta preferibili nello scritto, in particolare in poesia”, "uso più forme a seconda del contesto e delle finalità, se espositive o narrative; le forme accentate spesso hanno origine da contesti narratologici lontani nel tempo, risalen-

I3 Cito le annotazioni come sono state poste; tutti i commenti sono anche da consultare nel file di cui nella nota n. I I. 
ti per me a narrativa infantile antica o fiabesca";

5) la scelta delle forme a seconda del significato; in tal modo mi è stato suggerito di distinguere tra

a) "risolse un problema" ma "risolvette (decise) di andarsene via",

b) "assolvette a un obbligo" ma "assolse un imputato";

c) "si connesse ad internet; connetté il cavo alla stampante"

d “[...] e, a quanto disse, gli apparve la Madonna" ma "da tanto lo cercavo, e mi apparì, così all'improvviso che mi fece paura"; tale differenza non viene notata dai dizionari, dunque si tratta piuttosto di caratteristiche individuali che, però, possono essere oggetto di ulteriori interessanti studi.

Le tavole di coniugazione insieme ai dizionari dei verbi italiani, in quanto fonti cui si ricorre più spesso per verificare una flessione verbale incerta, dovrebbero prendere in considerazione tutti i problemi appena esposti. Oltre a una semplice registrazione delle forme flesse bisognerebbe, quindi, indicare in maniera esplicita le voci arcaiche o limitate a un certo contesto d'uso, i verbi che cambiano paradigmi flessivi se sono polisemici o verbi difettivi del Passato Remoto. Anche le dette tavole richiedono una costante revisione e aggiornamenti, perché - come scrive Giuseppe Antonelli ne La lingua in cui viviamo - "la grammatica di una lingua non è mai statica: è sempre in equilibrio dinamico tra norma e uso". ${ }^{14}$

\section{Povzetek}

$\mathrm{V}$ italijanskem sistemu glagolskih paradigem je za glagolski čas passato remoto značilno veliko število dvojnih ali celo trojnih alternativnih spregatev. Posledično v desetih spregatvenih tabelah in slovarjih, ki so bili objavljeni v Italiji, najdemo 38 glagolov z več spreganih oblik (pravilnih in nepravilnih). Namen pričujočega članka je preučiti

I4 Giuseppe Antonelli, La lingua in cui viviamo. Guida all'italiano scritto, parlato, digitato (Milano: BUR Rizzoli), 38.
I) kako so vse te oblike predstavljene v različnih spregatvenih tabelah in slovarjih glagolov (raziskavo zato omejujem samo na tiste glagole, ki imajo obe: pravilno in nepravilno obliko);

2) katere izmed njih se bolj pogosto uporabljajo v pisanih besedilih oz. kakšna je njihova dejanska uporaba v največjih korpusih pisane italijanščine (katere oblike predstavljajo visok delež vseh pojavljajočih se oblik posameznega glagola v času passato remoto) in

3) katere oblike rojeni govorci italijanščine danes raje uporabljajo (glagoli z dvema spregatvama: med glagoli na-ire se pogosteje uporablja pravilna spregatvena paradigma, med tistimi na -ere pa nepravilna oblika; glagoli s tremi spregatvenimi oblikami: med glagoli na -ere so bolj pogoste pravilne oblike, medtem ko za glagola apparire in comparire se bolj uporabljata obliki apparve in comparve, čeprav se pojavljata tudi s končnicami pravilne spregatve).

Spregatvene tabele in ostalo didaktično gradivo, ki predstavlja italijanskiglagolski sistem, bi morali expressis verbis nakazati katere so najpogostejše uporabljane glagolske oblike, kakšen je njihov kontekst uporabe (sploh če je ta omejen), katere oblike so danes arhaične in kateri glagoli ne razpolagajo z obliko za passato remoto.

\section{Abstract}

In the Italian verbal system, the passato remoto is distinguished by a particular abundance of double, or even triple, alternative inflections. Thus in Io different conjugation tables and dictionaries published in Italy we can find 38 verbs with more conjugated forms (regular and irregular). In this article, I attempt to examine

I) how these inflected forms are presented in several conjugation tables and Italian verb dictionaries (I limit my analysis to verbs that have both regular and irregular forms);

2) which forms are used more often in written texts, i.e., what is their real use in the largest corpora of written Italian (forms that constitute a relatively high percentage of all the occurrences of the passato remoto); and 3) which forms are preferred today by native Italian speakers (for the verbs that register two inflectional forms, verbs ending in -ire prefer the regular inflectional paradigm, those in -ere prefer irregular forms; verbs with three inflections, those in -ere prefer regular forms, while the verbs apparire and comparire prefer 
the forms apparve and comparve even though their regular endings are frequently used).

Conjugation tables and other didactic material that present Italian verbal system should indicate expressis verbis which are the most commonly used conjugated verbal forms, what is their context of use (if limited), which forms are archaic or lack the form for Passato Remoto.

\section{Bibliografia}

Tavole di coniugazione e dizionari dei verbi analizzati: vedi Tabella I.

Antonelli, Giuseppe. La lingua in cui viviamo. Guida all'italiano scritto, parlato, digitato. Milano: BUR Rizzoli, 2017.

Cresti, Emanuela, and Alessandro Panunzi. Introduzione ai corpora dell'italiano. Bologna: il Mulino, 2013

Della Valle, Valeria, and Giuseppe Patota. Ciliegie o ciliege? e altri 2406 dubbi della lingua italiana. Milano: Sperling \& Kupfer, 2012.

Gheno, Vera. «Sul digramma gn e sulla presen$z$ a della $i$ in forme verbali come guadagniamo», Redazione Consulenza Linguistica dell'Accademia della Crusca, 2005.

Novelli Silverio. Si dice? Non si dice? Dipende. L'italiano giusto per ogni situazione. Roma-Bari: Laterza, 2015 .

Stapek, Daniel. «Doppie forme regolari del Passato Remoto: desinenze -ei, - $\dot{e},-$ erono vs etti, -ette, -ettero», to appear. 